\title{
Research
}

\section{Individual differences in dopamine efflux in nucleus accumbens shell and core during instrumental learning}

\author{
Jingjun Cheng and Matthijs G.P. Feenstra ${ }^{1}$ \\ Netherlands Institute for Brain Research, 1105 AZ Amsterdam, The Netherlands
}

\begin{abstract}
Combined activation of dopamine D1- and NMDA-glutamate receptors in the nucleus accumbens has been strongly implicated in instrumental learning, the process in which an individual learns that a specific action has a wanted outcome. To assess dopaminergic activity, we presented rats with two sessions (30 trials each) of a one-lever appetitive instrumental task and simultaneously measured dopamine efflux in the shell and core accumbens subareas using in vivo microdialysis. Dopamine efflux was increased during each session in all areas. The behavioral performance of the rats in the second session led us to divide them into a learning group ( $>90 \%$ correct trials) and a nonlearning group. In the first session, the rats of the learning group showed significantly higher increases. The difference was most pronounced in the shell. In the second session, the dopamine increase was similar in both groups, although the learning groups now pressed the lever about three times more often and consequently obtained more rewards. We conclude that task-related activation of dopamine efflux is different between learning and nonlearning rats only during the learning phase. These results support the pharmacological evidence that dopamine is of particular importance during the instrumental learning process.
\end{abstract}

In instrumental learning, a subject acquires the knowledge that an action results in a wanted outcome. For instance, when rats learn to press a lever to obtain a reward, they learn about the contingency of action and outcome and about the outcome as a wanted goal, i.e., they acquire goal-directed behavior (Balleine and Dickinson 1998). Associative mechanisms controlling such behavior include Pavlovian conditioning, contingency learning, and habit formation (Robbins and Everitt 1996; Kelley 2004).

The neurobiological substrate of appetitive instrumental learning has not been fully disclosed yet, although recent research suggests that activation of NMDA-glutamate receptors is needed in a distributed network of prefrontal cortex (PFC), nucleus accumbens (NAC), and amygdala (Baldwin et al. 2000). The dopaminergic (DA) system is involved as well; coactivation of NMDA- and dopamine $\mathrm{D}_{1}$-receptors in the NAC core subarea and the medial PFC is required for learning (Smith-Roe and Kelley 2000; Baldwin et al. 2002b). What these studies have also suggested is that activation of $\mathrm{D}_{1}$-receptors is needed for performance of instrumental behavior as a blockade of these receptors after acquisition severely impaired behavior (Smith-Roe and Kelley 2000; Baldwin et al. 2002b). Salamone et al. (2003) came to a similar conclusion and maintain that accumbens DA is involved in behavioral activation and in facilitation of resources to work toward a goal. Using microdialysis measurements, these researchers showed that DA efflux is activated during performance of instrumental behavior (McCullough et al. 1993; Sokolowski et al. 1998). In contrast, DA efflux in the medial PFC was reported not to increase during performance of a lever-press task, but exclusively during acquisition (Izaki et al. 1998).

DA measurements in NAC during instrumental learning have not been reported; therefore, we decided to determine DA efflux during the acquisition of lever-press behavior and to apply a similar approach as we did in a previous study (Cheng et al.

'Corresponding author.

E-mail M.Feenstra@nih.knaw.nl; fax 31-20-6961006.

Article and publication are at http://www.learnmem.org/cgi/doi/10.1101/ Im.1806.
2003), targeting both subareas, shell and core, and relating behavioral performance with measurements of DA efflux during two sessions of instrumental learning.

\section{Materials and Methods}

\section{Subjects}

All experiments were approved by the Animal Experimentation Committee of the Royal Netherlands Academy of Arts and Science and were carried out in agreement with Dutch laws (Wet op de Dierproeven, 1996) and European regulations (Guideline 86/ 609/EEC). Twenty-eight male rats from a Wistar-derived strain (Harlan/CPB) were socially housed under a reversed day/night cycle (white light from 7:00 p.m. to 7:00 a.m., diminished red light from 7:00 a.m. to 7:00 p.m.). They were kept for at least 1 wk with food and water ad libitum. The animals were experimentally naive and were handled daily.

\section{Surgery}

Starting on the day of surgery, animals (now weighing about $250 \mathrm{~g})$ were individually housed in Perspex cages $(25 \times 25 \times 32 \mathrm{~cm})$. Rats were anesthetized with intramuscular Hypnorm $(0.24 \mathrm{mg} / \mathrm{kg}$ fentanyl citrate and $7.5 \mathrm{mg} / \mathrm{kg}$ fluanisone, Janssen) and subcutaneous Dormicum $(0.75 \mathrm{mg} / \mathrm{kg}$ midazolam, Roche). A microdialysis probe (active membrane length $2 \mathrm{~mm}$ ) was placed in the NAC shell $(A+1.7 \mathrm{~mm}$; L-0.8 mm from bregma and V-8.5 mm from skull surface) or core (A $+1.7 \mathrm{~mm}$; L-1.8 $\mathrm{mm}$ from bregma and $\mathrm{V}-8.0 \mathrm{~mm}$ from skull surface) as described before (Cheng et al. 2003). Subcutaneous Finadyne $(50 \mathrm{mg} / \mathrm{kg}$ flunixin meglumide, Schering-Plough) was given as a post-surgical analgetic. After recovery from anesthesia, each rat was returned to its individual cage with free access to food and water. Three days later, experiments were started by removing all food from the cage at the end of the afternoon before the testing day.

\section{Behavioral and neurochemical apparatus}

Instrumental learning and testing was conducted in Skinner boxes (MED Associates), mounted within sound and lightattenuating chambers, and dimly illuminated by a light oriented 
toward the ceiling. Through an opening in the ceiling, the microdialysis probes on the subject's head could be connected to the outside swivel. The Skinner box was equipped with a food dispenser, where reward pellets (Noyes, formula P, $45 \mathrm{mg}$; Sandown Scientific) could be delivered, and with two levers and two lights above them. Because of the extended stay in the box, a water bottle was present. Instrumental learning was tested in two sessions of 30 trials each. Each trial started when one of the levers came out into the box and the light above the lever was turned on. If the rat pressed the lever within $112 \mathrm{sec}$, the lever retracted, the light was turned off, and a pellet was delivered. The next trial always started $112 \mathrm{sec}$ after the start of the previous one, and the length of intertrial intervals were adapted to that. This is because we scheduled two trials in one microdialysis sample period (3.75 min). When the lever was not pressed, it was retracted and the light was turned off, but no pellet was delivered, and the next trial was started. Instrumental performance was monitored by recording the number of lever presses. In addition, nose pokes into the food dispenser were recorded by an infrared photobeam.

The microdialysis set-up was as described previously (Cheng et al. 2003) The lag-time between dialysis cannula and HPLC injector was about $15 \mathrm{~min}$. The HPLC set-up was somewhat adapted; pulse dampener, injector (5- $\mu \mathrm{L}$ loop), column, and detector cell (operated at $+280 \mathrm{mV}$ ) were now kept at $40^{\circ} \mathrm{C}$.

\section{Experimental procedures}

On the day of testing, the rats were introduced in the Skinner box, where five pellets were present in the food tray, and connected with the online microdialysis set-up. The rats had no previous experience in any experimental box. Animals were left undisturbed for at least $2 \mathrm{~h}$, during which time dialysis samples were automatically injected every $3.75 \mathrm{~min}$ on the column and analyzed online. When the levels of DA had stabilized, the first instrumental learning session was started, consisting of 30 trials (the time of two trials is $3.75 \mathrm{~min}$ for one sample of measurement). This was followed by an intersession interval of about $2 \mathrm{~h}$ before the second session started. Following this session, samples were taken until the DA levels had stabilized again.

\section{Experimental procedures additional experiment}

An additional experiment was carried out using animals with probes placed in the NAC shell. The only difference from the main experiment was in the first four trials of the instrumental learning program; when the lever was not pressed in these first four trials, a reward pellet was delivered at the end of the trial period (i.e., $112 \mathrm{sec}$ ). This was the case in both the first and second session. This program was used because is has been the standard paradigm for rats for a number of years to learn lever pressing in Skinner boxes.

\section{Probe placement verification}

After completion of the experiments, the rats were euthanized by inhalation of a mixture of $\mathrm{CO}_{2} / \mathrm{O}_{2}(70 / 30)$, followed by $100 \%$ $\mathrm{CO}_{2}$. The probes were removed and brains were taken out of the skull and frozen at $-20^{\circ} \mathrm{C}$. Coronal $(20-\mu \mathrm{m})$ cryostat sections were stained with thionine and examined with the microscope for the precise location of the microdialysis probes.

\section{Data analysis}

\section{Behavioral results}

The behavior of all rats was assessed by analysis of the number of lever presses (pellets obtained) and the number of nose pokes during a sample period (= two trials). From these, the total numbers of lever presses and nose pokes for each session $(=15$ samples or 30 trials) were calculated. The two behavioral sessions coincided with the microdialysis sample periods 5-19 (session 1) and 37-51 (session 2) (see Figs. 2 and 3 below). On the basis of the performance in the second session, the rats were divided into a learning group and a nonlearning group. The criterion for inclusion in the learning group was a response rate of $90 \%$ or more (i.e., 27 or more lever presses or pellets obtained). As the purpose of the experiments was to analyze and compare behavior and DA efflux for both areas, an overall analysis was always followed by a separate analysis of the data from rats with microdialysis probes in the shell or in the core. When behavioral analysis indicated group differences, these groups were also always separately analyzed for DA efflux.

The lever press data were analyzed using nonparametric tests. This is because only three outcomes were possible $(0,1$, or 2 lever presses per sample period). The numbers of lever presses per sample period were analyzed separately for each session by repeated measures analysis (Friedman's ANOVA) with sample as within-subject factor. Total numbers per session were analyzed in a similar way using the Wilcoxon test. Between-group comparisons were carried out for lever presses per sample period or per session (Kruskal-Wallis test or Mann-Whitney test, depending on the number of groups; in both cases, the exact significance was used).

The nose-poke data were analyzed using repeated measures ANOVA. The degrees of freedom were corrected using the Huynh-Feldt method. Between subjects, factors were area and group (two levels: core and shell; learning and nonlearning) and sample was the within-subjects factor. Statistical analyses of these data were carried out separately for the two sessions. An overall analysis was always followed by a separate analysis of the data from rats with microdialysis probes in the shell and in the core. When significant within-subject main effects or interaction effects were obtained, nose-poke numbers for sample periods 6-19 (session 1) or 38-51 (session 2) were compared with the first sample (5 or 37 , respectively) (simple contrasts in repeated measures ANOVA). As multiple comparisons are carried out here, a more conservative level of significance was chosen $(P<0.01)$. Total numbers of nose pokes for a session were analyzed in a similar way using sessions as the within-subjects factor and were followed by post hoc comparison of session 1 vs. 2 when indicated. When significant between-subject main effects or interaction effects were obtained, ANOVA followed by StudentNewman-Keuls (SNK) test or independent samples $t$-test were used to assess group differences.

To further analyze differences in behavior, nonparametric ranking analysis was carried out on the sample periods in which the rats obtained the first pellet, obtained two pellets for the first time, or obtained the last pellet in that session.

\section{Neurochemical results}

Extracellular DA levels were calculated as percentage of baseline, with the four samples immediately preceding each behavioral session serving as the $100 \%$ level. Maximum increases in DA efflux (DA-max $=$ highest concentration expressed as percentage of baseline measured for each rat during the session) and total DA efflux (DA-auc, area-under-the-curve $=$ sum of percent increases for all samples for each rat during the session) were determined for each session

The neurochemical data were analyzed using repeated measures ANOVA. The degrees of freedom were corrected using the Huynh-Feldt method. Between-subjects factors were area and group (two levels: core and shell; learning and nonlearning) and sample was the within-subjects factor. Statistical analyses of these data were carried out separately for two sessions. An overall analysis was always followed by a separate analysis of the data from rats with microdialysis probes in the shell and in the core separately. When significant within-subject main effects or interaction effects were obtained, DA concentrations for sample periods 5-30 (session 1) or 37-51 (session 2) were compared with those of all four baseline samples (1-4 or 33-36, respectively). A sample concentration is reported as significantly different from baseline only when significance was reached in all four comparisons. When significant between-subject main effects or interaction effects were obtained, Student-Newman-Keuls (SNK) test or independent samples $t$-test were used to assess group differences.

DA-max and DA-auc for a session were analyzed in a similar way using session as the within-subjects factor and area and 
group as between-session factor and were followed by post hoc comparisons when indicated or planned.

\section{Correlations}

Correlations between behavioral and neurochemical data were studies using Pearson's (DA concentration vs. nose pokes) or Spearman's (DA concentration vs. lever press) correlation coefficients.

\section{General}

Statistical significance was set at a probability level of $P<0.05$ for all tests (except where otherwise indicated). All statistical tests were performed using the SPSS 11.0 package (SPSS).

\section{Results}

\section{Dialysis probe locations}

Figure 1 illustrates the location of the probes within the NAC shell and core. Data from rats with incorrectly placed probes are not reported. The final analysis includes 28 rats, in four groups, i.e., nonlearning shell and core groups ( $n=6$ each) and learning shell and core groups ( $n=8$ each).

\section{Behavioral results}

Most of the rats started to press the lever in one of the first trials of the first session. Apart from one rat in the first session, all rats pressed the lever and obtained pellets in both sessions. Analysis of the lever-press data showed a normal distribution (ShapiroWilk test). In the second session, the data were not normally distributed (Shapiro-Wilk statistic $=0.781 ; P<0.001$ ). Part of the data were clustered at values of 27,29 , and 30 lever presses per session, but the rest was normally distributed. We adopted a criterion for learning of $90 \%$ correct (i.e., 27 lever presses). Sixteen of the 28 rats tested $(57 \%)$ reached this criterion and they are referred to as the learning group.

\section{Total lever presses}

The shell and core learning groups showed a significant increase in total lever presses from the first to the second session (Wilcoxon: $\mathrm{Z}=-2.524 ; P<0.01$ for both). This indication of instrumental learning was absent in the nonlearning groups. In session 2 , both learning groups had significantly higher numbers of total lever-presses than the nonlearning groups (Kruskal-Wallis $\chi^{2}=20.768, P<0.001$; followed by Mann-Whitney $\mathrm{Z}=-3.147$, $P<0.001$ (shell) and $-3.176, P<0.001$ (core). In session 1 , this difference was significant only for the shell group (Kruskal-Wallis $\chi^{2}=9.307, P<0.05$; Mann-Whitney $\mathrm{Z}=-2.324, P<0.05$ for the shell) (Table 1).

\section{Lever presses per sample}

The within-subjects factor sample was never significant for any group, neither in session 1 nor in session 2 (Friedman's ANOVA). The between-subject factors were first tested using Kruskal-Wallis ANOVA of the four groups (shell and core, learning and nonlearning). Significant differences were obtained in sample periods 8 and $18\left(\chi^{2}=7.974\right.$ and 9.132 , both $\left.P<0.05\right)$ and 15 and 16 $\left(\chi^{2}=10.869\right.$ and 10.587 , both $\left.P<0.01\right)$ of session 1 and all (3751, $\chi^{2}$ between 12.793 and 22.338; all $P \leq 0.001$; except 41 : $\chi^{2}=9.734, P<0.05$; and $\left.47: \chi^{2}=10.522, P<0.01\right)$ sample periods of session 2. Subsequent Mann-Whitney tests were used on these sample periods to compare learning and nonlearning groups of either shell- or core-implanted animals. The results (Figs. 2, 3) indicate that the learning groups had more lever presses in two of the 15 sample periods in the first session (shell samples 15 and $16: Z=-2.560$ and -2.258 ; core samples 16 and 18: $Z=-2.258$ and -2.076 , all $P<0.05)$, but in almost all sample periods (shell: 14 of 15; core: 13 of 15) in the second session in both subgroups ( $\mathrm{Z}$ between -2.266 and -3.483 ; $P<0.01$ in general).

In the second, but not the first session, the learning groups made their first lever press at an earlier stage of the session than the nonlearning groups, both in the shell and in the core groups (Kruskal-Wallis $\chi^{2}=16.656$ and 22.055 for the first sample period with one or two presses, respectively).

\section{Total nose pokes}

Overall analysis of the total number of nose pokes per session produced a significant effect of session $\left(F_{(1,24)}=6.131, P<0.05\right)$ (Table 1), indicating an increased level of approach behavior toward the food dispenser in the second session. In addition, a significant effect of group (learning vs. nonlearning; $\left.F_{(1,24)}=19.348, P<0.001\right)$, but not of area, was obtained. Subsequent analysis indicated that the rats in the shell and the core learning groups made more nose pokes in both the first and second session when compared with the nonlearning groups (ANOVA session 1: $F_{(3,27)}=6.821, P<0.01$; session 2: $F_{(3,27)}=11.177, P<0.001$; followed by SNK tests). Upon separate analysis of all groups, the within-subjects factor was not significant.

\section{Nose pokes per sample}

When results for separate sample periods (Figs. 2, 3) were analyzed, a main effect of sample was present for the first session $\left(F_{(14,336)}=2.991, P<0.01\right)$, with an interaction (sample $\mathrm{x}$ group, $\left.F_{(14,336)}=2.316, P<0.05\right)$ and a between-subjects effect of group $\left(F_{(1,24)}=20.318, P<0.001\right)$, but not of area. Upon separate analysis, the withinsubjects factor sample was significant only in the learning groups (shell $F_{(14,98)}=2.318, P<0.05 ;$ core $\left.F_{(14,98)}=3.715, P<0.001\right)$. This indicates an increase in reward-related approach activity in the course of this first session in the learning groups only. The sample periods in which the number of nose pokes was significantly higher $(P<0.01)$ than that in the first one (sample 5) are indicated by closed symbols in Figures 2 and 3 . For the second session, an interaction (sample $\mathrm{x}$ group, $F_{(14,336)}=2.086$ $P<0.05)$ and a between-subjects effect of group $\left(F_{(1,24)}=32.266, P<0.001\right)$ was observed, but not a within-subjects effect or a between-subjects effect of area. Upon separate analysis, the within-
Figure 1. Schematic representation of the location of the microdialysis probe within the nucleus accumbens core and shell (for both $n=14$ ). Coronal sections of the brain are shown at different levels before bregma (Reprinted with permission from Elsevier ( 1997, Paxinos and Watson 1997).

\section{Learning \& Memory} www.learnmem.org 
Table 1. Summary of the neurochemical and behavioral results

\begin{tabular}{|c|c|c|c|c|c|c|c|c|}
\hline & \multicolumn{2}{|c|}{ DAauc } & \multicolumn{2}{|c|}{ DAmax } & \multicolumn{2}{|c|}{ Lever presses } & \multicolumn{2}{|c|}{ Nose pokes } \\
\hline & Session 1 & Session 2 & Session 1 & Session 2 & Session 1 & Session 2 & Session 1 & Session 2 \\
\hline SHELL Learning (8) & $409.0 \pm 50.2$ & $203.4 \pm 17.4^{\mathrm{b}}$ & $149.8 \pm 4.6$ & $132.6 \pm 2.8^{\mathrm{b}}$ & $18.6 \pm 2.0$ & $29.3 \pm 0.4^{\mathrm{b}}$ & $375 \pm 47$ & $462 \pm 23$ \\
\hline Nonlearning (6) & $161.5 \pm 38.2^{\mathrm{a}}$ & & $125.1 \pm 4.0^{\mathrm{a}}$ & $124.0 \pm 3.2^{c}$ & $8.3 \pm 3.0^{\mathrm{a}}$ & $10.7 \pm 2.9^{\mathrm{a}}$ & $131 \pm 33^{a}$ & $240 \pm 6$ \\
\hline $\begin{array}{l}\text { SHELL Learning (7) (addit. } \\
\text { exp.) } \\
\text { Nonlearning (6) (addit. }\end{array}$ & $331.5 \pm 36.5$ & $161.8 \pm 25.2^{\mathrm{b}}$ & $148.9 \pm 5.3$ & $126.1 \pm 3.4^{b}$ & $22.9 \pm 2.3$ & $29.9 \pm 0.1^{b}$ & $493 \pm 46$ & $388 \pm 44$ \\
\hline $\begin{array}{l}\text { exp.) } \\
\text { CORE Learning ( } 8) \\
\text { Nonlearning }(6)\end{array}$ & $\begin{array}{l}174.3 \pm 29.2^{\mathrm{a}} \\
350.6 \pm 33.2 \\
213.5 \pm 34.2^{\mathrm{a}}\end{array}$ & $\begin{array}{l}215.5 \pm 61.7 \\
299.0 \pm 34.3 \\
227.1 \pm 18.0\end{array}$ & $\begin{array}{l}131.0 \pm 3.4^{a} \\
141.5 \pm 3.8 \\
134.6 \pm 4.8\end{array}$ & $\begin{array}{l}130.6 \pm 5.7 \\
137.6 \pm 4.8 \\
141.9 \pm 3.3\end{array}$ & $\begin{array}{l}11.2 \pm 2.3^{\mathrm{a}} \\
17.6 \pm 2.8 \\
10.2 \pm 2.4\end{array}$ & $\begin{array}{l}13.2 \pm 1.3^{\mathrm{a}} \\
29.4 \pm 0.4^{\mathrm{b}} \\
10.8 \pm 1.4^{\mathrm{a}}\end{array}$ & $\begin{array}{l}199 \pm 66^{a} \\
359 \pm 61 \\
153 \pm 38^{a}\end{array}$ & $\begin{array}{l}192 \pm 19^{\mathrm{a}} \\
506 \pm 60 \\
174 \pm 42^{\mathrm{a}}\end{array}$ \\
\hline Nonlearning (6) & $213.5 \pm 34.2^{\mathrm{a}}$ & $227.1 \pm 18.0$ & $134.6 \pm 4.8$ & $141.9 \pm 3.3$ & & $10.8 \pm 1.4^{\mathrm{a}}$ & $153 \pm 38^{a}$ & $174 \pm 42^{a}$ \\
\hline
\end{tabular}

Total DA efflux (area-under-the-curve, DAauc), maximal DA efflux (DAmax), total numbers of lever presses (=pellets obtained), and nose pokes in Sessions 1 and 2 for the different groups. Values are means ( \pm SEM).

aSignificantly different from the Learning group.

bSignificantly different from Session 1.

cSignificantly different from CORE Nonlearning.

subjects factor sample was significant only in the shell learning group $\left(F_{(14,98)}=3.529, P<0.01\right)$. The number of nose pokes in sample 37 was lower than that in the first one, indicated by a closed symbol.

As a follow-up to the significant group and groupinteraction effects, the number of nose pokes of the learning and nonlearning groups were compared separately for the shell and core. Differences in the number of nose pokes between learning and nonlearning groups obtained (independent samples $t$-test) are presented in Figures 2 and 3 for the shell and core. The results indicate that the learning groups had more nose pokes in the end of the first session and in the beginning of the second session in both subgroups.

\section{Neurochemical results}

Basal concentrations of DA

Basal levels of DA in microdialysates from the NAC shell $(n=14)$ and core $(n=14)$ group were $2.56 \pm 0.33$ and $2.25 \pm 0.26 \mathrm{pg} / 5 \mu \mathrm{L}$ (means $\pm \mathrm{SEM}$ ), respectively, and were not significantly different.

Effect of instrumental learning on DA efflux per sample

DA efflux increased during the first session and an overall analysis showed a main within-subjects effect of sample $\left(F_{(29,696)}=32.032, P<0.001\right)$, a main between-subjects effect of group $\left(F_{(1,24)}=22.576, P<0.001\right)$, but not of area and an interaction (sample $\mathrm{x}$ group: $\left.F_{(29,696)}=3.946, P<0.001\right)$. Figures 2 and 3 show the time curves of DA efflux in the shell and core of learning and nonlearning groups. Separate analysis of both areas resulted in a main effect of sample (shell: $F_{(29,348)}=14.619$, $P<0.001$; core: $F_{(29,348)}=18.479$, $P<0.001)$, a main effect of group (shell: $F_{(1,12)}=16.119, \quad P<0.01$ : core: $\left.F_{(1,12)}=6.880, P<0.05\right)$ and a sample $\times$ group interaction (only in shell: $\left.F_{(29,348)}=3.224, P<0.01\right)$ for the first session. All separate groups showed significant sample effects in session 1 (shell learning: $F_{(29,203)}=13.342, P<0.001$; shell nonlearning $F_{(29,145)}=4.24$,
$P<0.001$; core learning $F_{(29,203)}=15.916, P<0.001$; core nonlearning $\left.F_{(29,145)}=5.580, P<0.001\right)$. The samples taken during the session that had significantly higher DA levels than all four baseline samples are shown in Figures 2 and 3 as filled symbols. As a follow-up to the significant group and group-interaction effects, DA efflux of the learning and nonlearning groups were compared separately for the shell and core. The learning groups showed higher efflux than the nonlearning groups in samples 8 and 13-19 (shell) and in samples 9, 11, 16, 18, and 19 (core) (independent samples $t$-test).

During the second session, DA efflux was again increased and analysis of the results showed a main within-subjects effect of sample $\left(F_{(29,696)}=28.557, P<0.001\right)$, main between-subjects effects of group $\left(F_{(1,24)}=6.060, P<0.05\right)$ and area $\left(F_{(1,24)}=7.888\right.$, $P=0.01$ ) and an interaction (sample $\mathrm{x}$ area: $F_{(29,696)}=2.231$, $P<0.01)$. When analyzed for separate areas, within-subject ef-
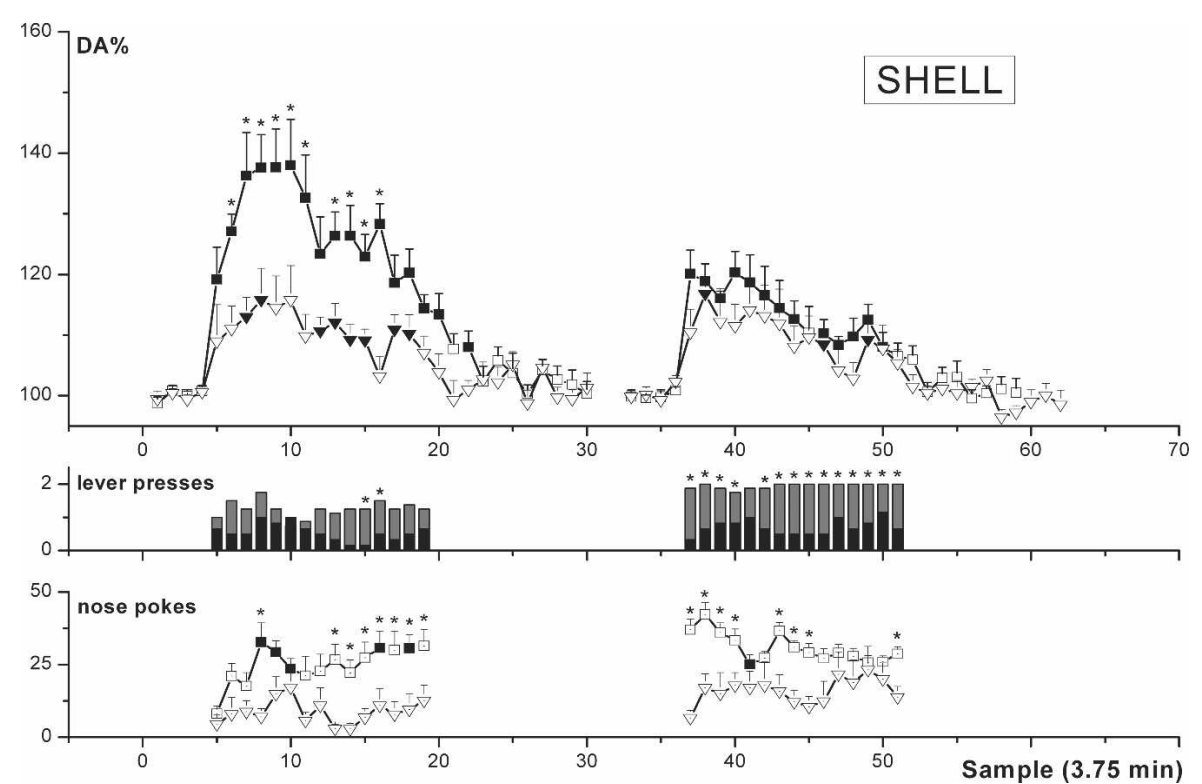

Figure 2. Mean changes in extracellular dopamine (DA) levels in the nucleus accumbens shell in the learning (squares) and nonlearning (inverted triangles) groups during two sessions of instrumental learning (top vertical axis). All concentrations are expressed as percentage of basal $\pm \mathrm{SEM}$. Filled symbols indicate significant differences compared with all four baseline samples $(P<0.05)$. Mean numbers of lever presses (= food pellets obtained) in two trials (one dialysis sample time) are given as bar graphs (middle vertical axis: gray bars = learning group; black bars = nonlearning group). Mean numbers of nose pokes in two trials are given in the bottom graphs. Filled symbols in the nose-poke graph indicate significant differences compared with the first sample $(P<0.01)$. (*) Significant differences between learning $(n=8)$ and nonlearning $(n=6)$ groups $(P<0.05)$. 

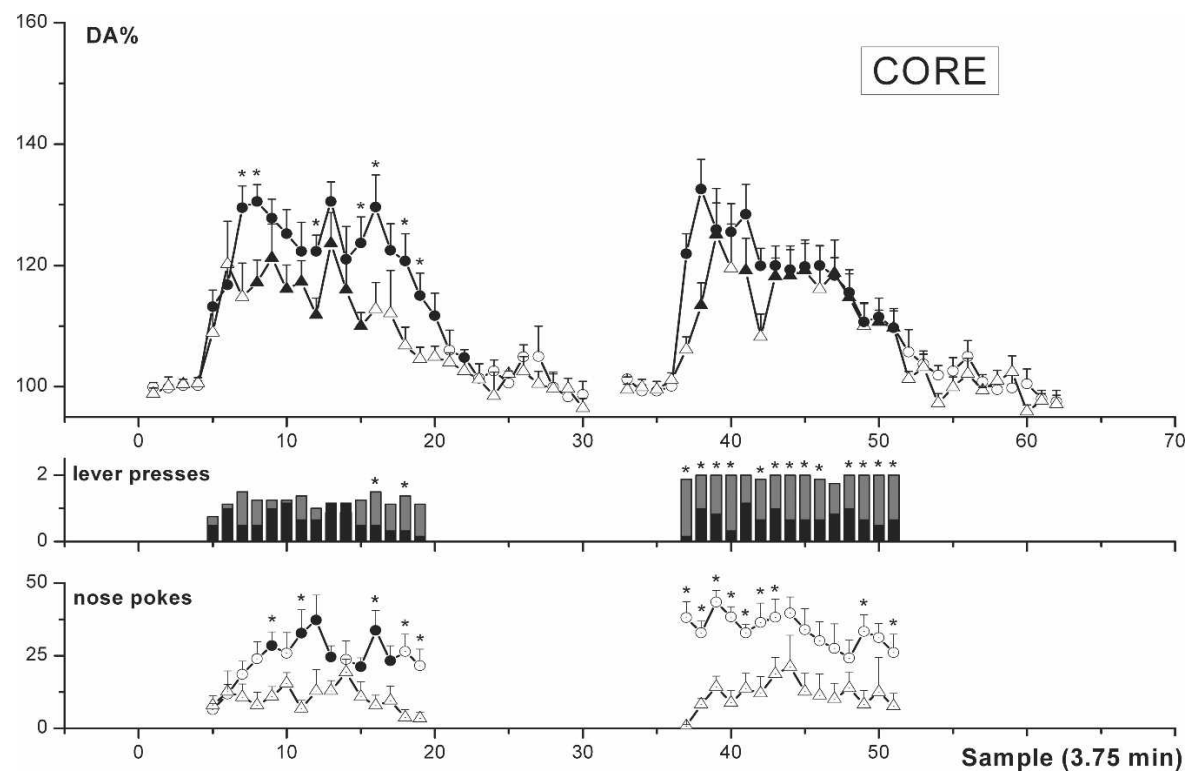

Figure 3. Mean changes in extracellular dopamine (DA) levels in the nucleus accumbens core in the learning (circles) and nonlearning (triangles) groups during two sessions of instrumental learning (top vertical axis). For further explanation, see Figure 2. significant session $x$ group $\left(F_{(1,24)}=6.709 P<0.05\right)$ interaction. Subsequent analysis indicated that both shell and core learning groups had higher DAauc than the respective nonlearning groups $\left(\right.$ ANOVA $F_{(3,27)}=7.831$, $P=0.001$, followed by SNK-test, $P<0.05$ ) during session 1 , but not in session 2. The shell learning group showed higher DAauc in session 1 compared with session $2\left(F_{(1,7)}=12.491, P=0.01\right)$.

The maximum DA increase (Table 1) was significantly different between groups $\left(F_{(1,24)}=8.995, P<0.01\right)$ with significant group $\mathrm{x}$ area $\left(F_{(1,24)}=6.580\right.$, $P<0.05)$, session $\mathrm{x}$ group $\left(F_{(1,24)}=4.322, P<0.05\right)$ and session $\mathrm{x}$ area $\left(F_{(1,24)}=6.746, P<0.05\right)$ interactions. DAmax in session 1 was significantly different between groups (ANOVA $\left.F_{(3,27)}=6.324, P<0.01\right)$. Subsequent SNK-tests showed a higher DAmax in the shell learning group than in the nonlearning group. The shell learning group showed higher DAmax in session 1 compared with session 2 $\left(F_{(1,7)}=9.998, P<0.05\right)$. fects were obtained: shell: $F_{(29,348)}=11.236, P<0.001$; core: $F_{(29,348)}=18.595, P<0.001$, but no effect of group. On the other hand, comparison of the learning groups revealed a significant effect of area $\left(F_{(1,14)}=4.842 ; P<0.05\right)$ and post hoc analysis indicated that DA was higher in core than in shell in several samples $(42: t=-2.398, P<0.05 ; 50: t=-2.506, P<0.05 ; 51$ : $t=-2.995, P<0.05)$. All separate groups showed significant sample effects in session 2 (shell learning: $F_{(29,203)}=8.541$, $P<0.001$; shell nonlearning $F_{(29,145)}=3.948, P<0.001$; core learning $F_{(29,203)}=16.391, P<0.001$; core nonlearning $\left.F_{(29,145)}=5.738, P<0.001\right)$. The samples taken during the session that had significantly higher DA levels than all four baseline samples are shown in Figures 2 and 3 as filled symbols.

Visual inspection of the curves of the learning groups in Figures 2 and 3 suggests that DA efflux is more strongly increased in the beginning of the session than at the end. We tested this by repeated measures ANOVA for the learning groups taking the fourth, fifth, or sixth sample during the first session (samples 8 , 9 , or 10) and the second, third, and fourth samples for session 2 (samples 38, 39, and 40) as baseline. The final three samples taken during session 1 had lower DA efflux in the shell compared with all three baseline samples. For session 2, samples 47, 50, and 51 had lower DA efflux in the shell compared with all three baseline samples, while the final sample had lower DA efflux in the core compared with all three baseline samples. This result indicates that in the course of the session, DA increases tend to decrease.

As a follow-up to the significant group and groupinteraction effects, DA efflux of the learning and nonlearning groups was compared separately for the shell and core. The learning groups showed higher efflux than the nonlearning groups in samples 37-40, 43-45, and 51 (shell) and samples 37-43, 49, and 51 (core) (independent samples $t$-test).

\section{Effect of instrumental learning on DA efflux per session}

The total DA efflux (area-under-the-curve; Table 1) was significantly different between sessions $\left(F_{(1,24)}=6.876, P<0.05\right)$ and between groups $\left(F_{(1,24)}=25.900, P<0.001\right)$ with a

\section{Relation between neurochemical and behavioral data}

Strong correlations between DA efflux and the number of lever presses were observed in the first five samples of the first session (Spearman 0.404, $P<0.001$ and $0.684, P<0.001$ for learning and nonlearning groups, respectively). The number of nose pokes was correlated with DA efflux as well (Pearson 0.514, $P<0.001$ and $0.629, P<0.001$ for learning and nonlearning groups, respectively). Both groups differed, however, in that the learning group correlations were less (lever presses) or absent in the remainder of the session, but in the nonlearning group, correlations were strong. This group difference recurred in the beginning of the second session, where strong correlations were found in the nonlearning group only (first five samples lever presses: Spearman $0.521, P<0.001$; nose pokes Pearson 0.456, $P<0.001$ ).

\section{Results of additional experiment}

The results of this experiment, in which the first four trials ended with delivery of a pellet when the lever was not pressed, were very similar to those of the main experiment and results will not be presented in the same detail (see Figure 4; Table 1). Seven of 13 rats $(54 \%)$ reached the criterion of $90 \%$ responses in the first session and were classified as the learning group.

The main difference was that the number of nose pokes in the learning group was high from the first sample on and did not increase or decrease afterward. Therefore, a significant difference between the number of nose pokes made by the learning and the nonlearning groups was present in the very first sample (ANOVA main effect of group $F_{(1,11)}=14.158 ; P<0.01$; $t$-test learning vs. nonlearning samples 5, 8-10, 12, 13: $P<0.01$; samples 11, 14; $P<0.05)$. Indeed, the only difference between the total scores for the two experiments (Table 1) was an interaction experiment session $\left(F_{(1,23)}=7.753 ; P<0.05\right)$ for the numbers of nose pokes, but post hoc within- or between-group analyses did not result in any significant difference.

\section{Results of further subdivision}

We made an attempt to further subdivide the learning and nonlearning groups (combining main and additional experiments, 

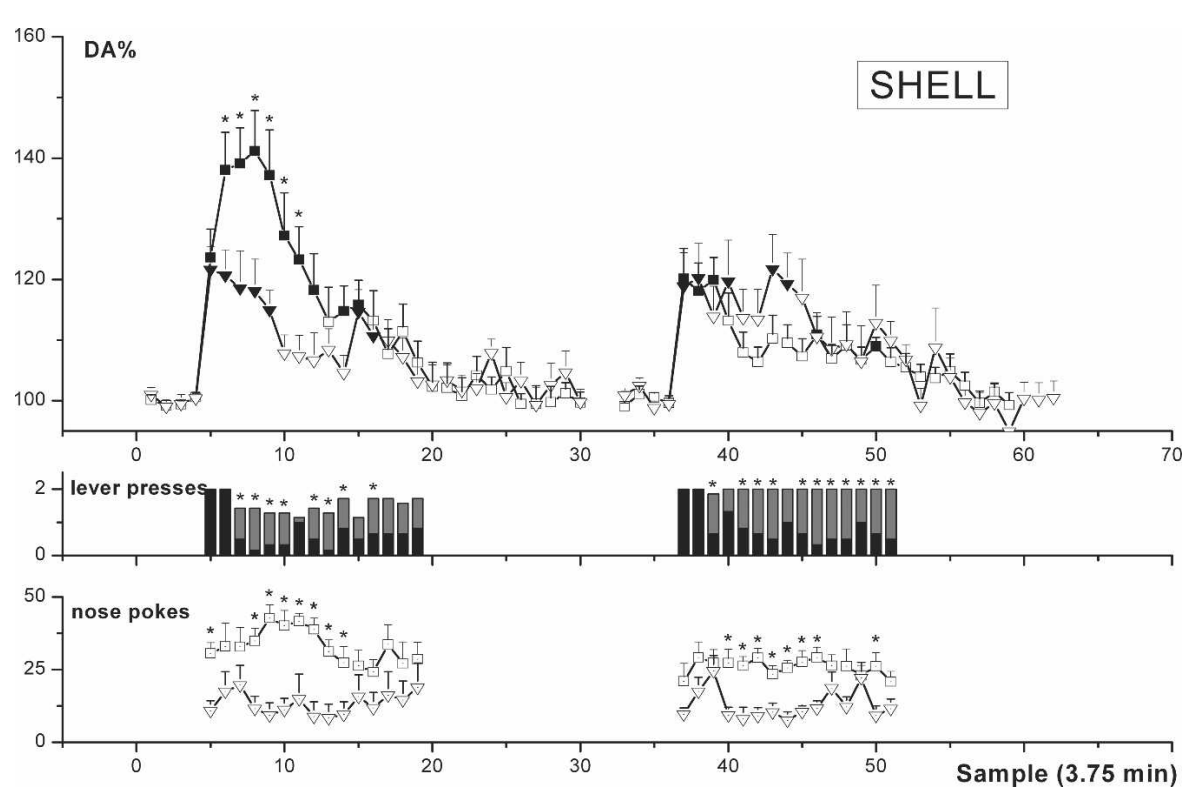

Figure 4. Mean changes in extracellular dopamine (DA) levels in the nucleus accumbens shell in the learning (squares) and nonlearning (inverted triangles) groups of the additional experiment during two sessions of instrumental learning (top vertical axis). The additional experiment differed from the main experiment in that a pellet was delivered when the animals did not press the lever in the available time in the first four trials. This was registered as a lever press. For further explanation, see Figure 2. learning and reached the criterion of $90 \%$ or more reward pellets obtained. These rats from the learning group showed higher task-related increases in DA efflux in the NAC during the first session than the rats that did not learn, and this difference was present both in the shell and in the core. Moreover, the rats from the learning group showed a higher increase in DA efflux in the NAC shell during the first session than during the second session. The results obtained in the NAC shell were confirmed in an independent experiment. These results suggest a relation between task-related increases in DA efflux and instrumental learning.

\section{Behavioral results}

We presented experimentally naive rats with a simple instrumental task; they had to press a lever to obtain a reward. Our experience is that almost all rats learn to perform the task with $90 \%$ $100 \%$ correct trials in the third or fourth session and that learning is not affected by the presence of microdialysis probes. The performance of the rats in the first shell and core) into two groups each. This subdivision was used for an analysis of the total lever presses, total nose pokes, and total DA efflux per session. Eight rats (two of the shell learning group; two of the core learning group; four of the additional shell learning group) pressed 26 or more times during the first session (87\%); these were classified as the "early learning" group. The other rats of the learning groups $(15$, six from shell and core groups and three from the additional shell learning group) pressed 21 times or less (with a mean of 15, i.e., 50\%), these were classified as the "normal learning" group. Of the nonlearning rats, five pressed between seven and 13 times more in the second than in the first session, while the others pressed less than in the first session or only one or two times more. These five rats (three of the shell nonlearning group, one of the core group, one of the shell additional group) were classified as the "late learning" group. The remainder (three of the shell nonlearning group, five of the core group, and five of the shell additional group) were classified as "not learning."

Both the normal learning and the early learning groups showed an increase in lever presses from sessions 1 to 2 (Wilcoxon $\mathrm{Z}=-3.416 ; P<0.001$ and $-2.539 ; P<0.01$, respectively) (Fig. 5). The late-learning group pressed about three times as much in the second compared with the first session, but this did not reach significance $(Z=-2.041 ; P=0.063)$. While the normal and the late-learning groups showed an increase in nose pokes from session 1 to session $2\left(F_{(1,14)}=5.447 ; P<0.05\right.$; $F_{(1,4)}=87.363, P=0.001$, respectively), the early learning group had a lower number of nose pokes in the second session $\left(F_{(1,7)}=10.086, P<0.05\right)$.

Both the normal and the early learning groups showed a decrease in DA-auc from session 1 to session $2\left(F_{(1,14)}=11.078\right.$; $P<0.01 ; F_{(1,7)}=24.072, P<0.01$, respectively). The increase in the late learning group was not significant $\left(F_{(1,4)}=3.733\right.$, $P=0.126)$.

\section{Discussion}

About $60 \%$ of the rats significantly improved their lever-press performance from the first to the second session of instrumental session varied, but did not lead us to separate them into different groups. However, when the numbers of lever presses (pellets obtained) in the second session were analyzed, a clear difference between individual animals became apparent. About $60 \%$ of the rats now reached a response level of $90 \%$ or higher and showed a strong improvement in task performance compared with the first session. These rats learned the instrumental task in two sessions. The remainder (nonlearning group) did not reach the criterion, with the majority performing on the same level as they did before. They also waited longer to start pressing in the second session than the learning group. An additional experiment, where the rats received a "free" reward if they did not press the lever in the first four trials, gave comparable results and led to a similar difference in the outcome of learning.

While the task performance did not increase significantly within the course of the first session, it did improve from session to session. Hernandez et al. (2002) reported a protein-dependent consolidation of instrumental learning in the core of the NAC in the first $2 \mathrm{~h}$ after training. We suggest that the procedural memory for the lever-press task in the present experiments was consolidated in the interval of $2 \mathrm{~h}$ between the sessions, to be retrieved immediately at the start of the second session.

The number of nose pokes showed a significant increase in the course of the first session, but only in the rats from the learning group. These animals also made more nose pokes in the second than in the first session, except for the small (early learning) subgroup that already achieved an almost maximal instrumental performance in the first session. This outcome suggests that the number of nose pokes may reflect (an aspect of) instrumental learning. This is also indicated by the results of the additional experiment; only the rats that started with a high level of nose poking activity in the first sample period were able to learn the task in two sessions. Kelley et al. (1997) reported a betweensession increase in nose poking during instrumental learning and suggested that it was caused by acquired incentive motivation. Acquisition of incentive motivation or attribution of incentive salience refers to the process by which stimuli-predicting reinforcement may come to generate responses (Berridge and Robin- 

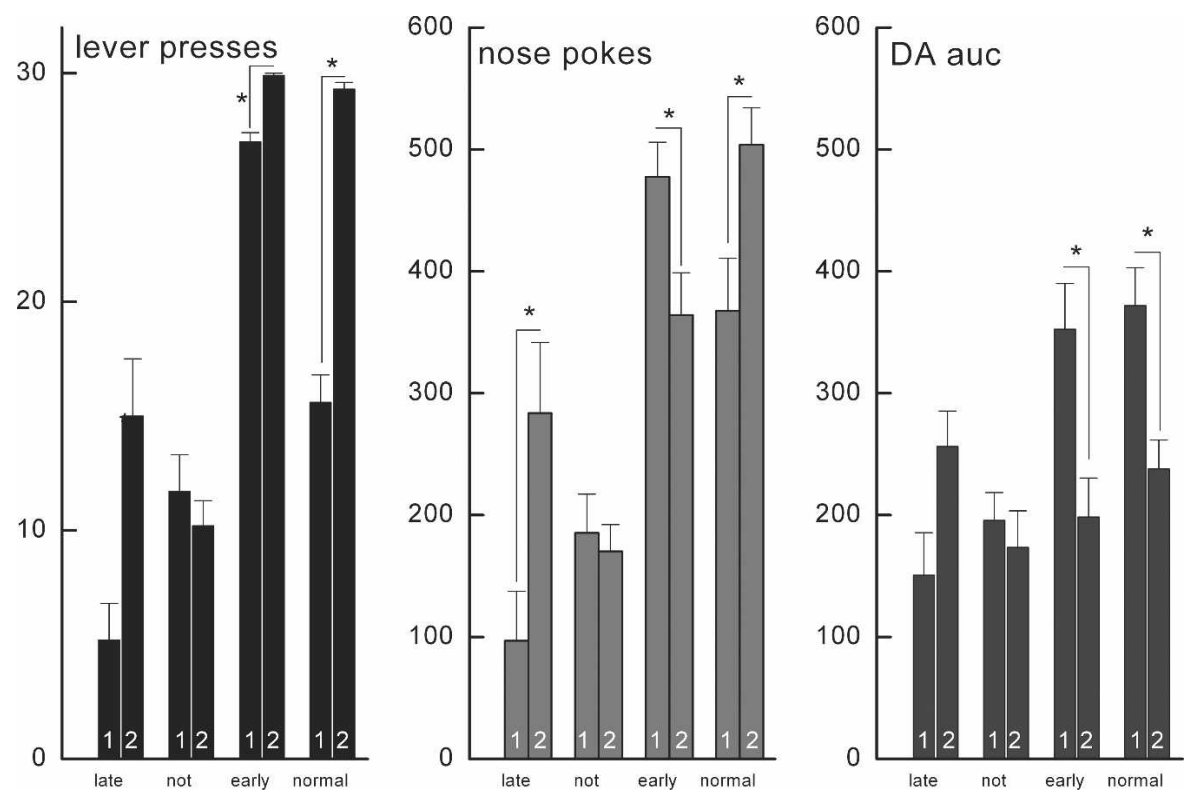

the core being critical for appetitive instrumental learning and mediation of motivational impact by conditioned stimuli, and the shell for integration of feeding behavior and mediation of motivational impact of unconditioned stimuli (Kelley 1999; Cardinal et al. 2002). The DA innervation of these subareas may have a differential role in these processes as well (Smith-Roe and Kelley 2000; Di Ciano et al. 2001; Cardinal et al. 2002; Di Chiara 2002). It was therefore of particular interest to follow DA changes in both areas. The basal levels and task-related increases of DA efflux were similar for shell and core groups in the first session. These results compare well with our previous study in which the DA responses during classical, appetitive conditioning were reported (Cheng et al. 2003). An interesting result was that the values for DAauc and DAmax in the shell learning group (in both the main and the additional experiment) decreased from session 1 to session 2, while the performance increased. This resembles the habituation of reward-induced effects in the shell, but not the core, as reported by Bassareo and

son 1998; Ikemoto and Panksepp 1999). Nose pokes (magazine entries) may be qualified (at least for a large part) as a Pavlovian approach behavior toward the food dispenser and reflect an aspect of instrumental learning that is distinct from both contingency learning and habit formation (Robbins and Everitt 1996; Dickinson et al. 2000; Parkinson et al. 2000; Kelley 2004).

At the start of the second session, the rats from the learning group had high rates of nose poking, but this tended to decrease in the course of the session (significance was reached only in the shell subgroup). Kelley et al. (1997) observed a between-session decrease in nose poking, once their rats reached a maximum level of lever-pressing performance. A similar decrease from session 1 to session 2 (Fig. 5) was present in our subgroup of early-learning animals. This suggests that (increased) nose poking may not only reflect (acquisition of) incentive motivation, but may also be related to the novelty of the task and the uncertainty about task organization and instrumental outcome that is typical for the early learning phase.

\section{Neurochemical results}

DA efflux in the NAC was elevated when the rats engaged in lever-pressing activities, confirming previous reports in which different measures of nerve terminal DA activity in vivo were used (Hernandez and Hoebel 1988; Salamone et al. 1989; Joseph and Hodges 1990; McCullough et al. 1993; Sokolowski et al. 1998; Roitman et al. 2004). As we observed a significant increase in DA efflux during both sessions in all groups and subgroups of rats, it may be concluded that DA efflux in the NAC is increased both during acquisition and performance of a simple leverpressing task. While this seems to be at odds with a previous report on DA efflux in the PFC (Izaki et al. 1998), our results do suggest a higher increase during the acquisition phase in the learning groups, which is in agreement with their findings.

The shell and core subareas are two distinct regions of the nucleus accumbens that have been suggested to have specific and contrasting functions in the regulation of motivated behavior,
Di Chiara (1997), although such habituation was stated to be present only in freely fed rats (Di Chiara 2002) and the animals in the present experiments were food restricted. The reverse, an increased DA response in the NAC core upon repeated exposure was also reported (Bassareo et al. 2002), but this was also obtained in freely fed rats only. Thus, it remains uncertain whether these effects of habituation and sensitization bear relevance to the outcome of our experiments.

As maximum values were generally reached in one of the first samples and increases were, at least in some cases, significantly lower at the end of the session, a within-session habituation was also apparent. This may reflect the long-lasting relative depression in release that was predicted and observed during extended periods of in vivo stimulation experiments (Montague et al. 2004).

Another remarkable finding is that once the rats had learned the task and had achieved a stable level of responding, they showed (in the second session) higher task-related increases in DA efflux in the core than in the shell. Therefore, we cannot confirm the higher responsiveness of DA in the shell during operant responding as suggested by Sokolowski et al. (1998).

In general, our results suggest that the differences in activation of DA projections to the shell and the core during instrumental learning are less than the differences in the reported consequences of DA activity in these areas. We came to a similar conclusion in our study of classical conditioning (Cheng et al. 2003).

\section{Relation between behavioral and neurochemical results}

The relation between the neurochemical measurements and the behavioral activities is complex. DA increases during appetitive behavior have been suggested to be related to presentation of reward, reward-related cues, and/or reward-related activities (Blackburn et al. 1992). In the second session of our experiments, these increases were similar (core) to those in the first session, or even less (shell), despite the fact that more lever presses were made and more pellets were obtained. Moreover, only in the first 
session did DA efflux increase more in the learning animals than in the nonlearning ones, although the difference in lever presses was much higher in the next session. Although this is suggestive evidence that the higher increases in DA efflux in the first session are not due to increases in presentation and consumption of rewards, it may not be conclusive, as it cannot be excluded that other factors such as habituation (at least in the shell, see above) might play a role. Most researchers will agree that DA efflux is activated during feeding, but different aspects of feeding may be more strongly associated with increased DA efflux than others. The outcome of many studies points to a strong relation between DA efflux and food-related stimuli and food-seeking behavior (Blackburn et al. 1992; Berridge and Robinson 1998; Ikemoto and Panksepp 1999; Redgrave et al. 1999; Salamone et al. 2003). The only studies that may provide an unequivocal answer to the question as to which part of feeding behavior in the context of an operant task shows a temporary relationship to increased DA efflux are those using subsecond measurements of DA efflux, e.g., in vivo fast-scan cyclic voltammetry. Using such a set-up, Roitman et al. (2004) observed strong increases by the presentation of incentive stimuli to well-trained animals, culminating in a maximum at the moment of the lever press. Subsequent intraoral reward presentation did not further increase DA, but coincided with a decrease to basal levels, suggesting that the food-seeking behavior is the major determinant of DA increases. Obviously, in the present experiments we did not achieve such a high temporal resolution and we can only compare efflux in different conditions and phases of the instrumental learning process. In addition, the animals in the present experiments were not trained, and the novelty of the situation should make an important difference (see further).

The number of nose pokes also increased from session 1 to session 2 in most animals of the learning groups, whereas the total DA efflux decreased, making it difficult to detect a relationship between nose pokes and DA efflux that is valid across the various groups.

Cues that predict reward presentation have been reported to activate DA neurons (Schultz 2002) and lead to increased DA efflux (Blackburn et al. 1992; Feenstra 2000; Joseph et al. 2003). We showed that reward-predicting stimuli increased DA efflux in NAC shell and core in classical conditioning (Cheng et al. 2003). However, while the responsiveness to the cues was increased in the learning group compared with the nonlearning group in the second session in our present study, this was not the case for DA efflux.

If differences in reward-related activities such as lever presses and nose pokes, in reward presentation or consumption, or in reward-predicting cues, do not provide a ready explanation for the differences in DA efflux, which parameter can? In electrophysiological studies, primate DA neurons show a learningrelated shift, from activation at reward presentation to activation at reward-predicting stimuli (Hollerman and Schultz 1998). The response to the predicting stimulus depends on the motivational state, i.e., the stimulus-acquired incentive motivational properties (Satoh et al. 2003). Recently, Pan et al. (2005) showed that decreased activation by reward delivery and increased activation by cue presentation occur simultaneously in rat DA neurons, so that early during training, both reward- and stimulus-induced responses are present. The combined effect on DA activity of the early trials may be stronger than that of the later trials, resulting in a decrease in effective total transmitter release in the course of training (Pan et al. 2005). Roitman et al. (2004), using fast-scan cyclic voltammetric measurements of NAC DA efflux, showed that animals that did not immediately press the lever after the cue presentation instead of a single phase of DA set off by cue presentation and peaking at the lever press, had two phases of increased DA, one after detecting the cue and a second, larger one, when pressing the lever. This situation will occur more frequently during learning. In addition to these phasic, eventrelated activations, DA neurons have also been reported to show longer-lasting activations during the expectancy phase in between stimulus and reward presentation when uncertainty about the outcome is high (Fiorillo et al. 2003). However, the strong increase in DA efflux during the task acquisition may also reflect the novelty of the situation. The novel presentation of the lever and, eventually, the reward may be expected to lead to substantial activation of DA activity and efflux (e.g., Feenstra and Botterblom,1996; Feenstra et al. 2000). The combination of the learning-related process of attribution of incentive salience (cf. Berridge and Robinson 1998) with novelty and uncertainty may lead to high increases in DA efflux during the first phase of instrumental learning.

We measured changes in DA efflux on a timescale (minutes) that is often regarded as reflecting changes in tonic, but not phasic, DA activity (Schultz 2002; Wightman and Robinson 2002). Yet, it is essentially unknown what the precise relation is between tonic (resolution in minutes), intermediate (seconds), and phasic (subsecond) DA modes of neuronal activity and release. Therefore, it is interesting to note that the patterns of increases we observe in tonic DA activity during learning apparently compare well with alterations in phasic neuronal activity (Hollerman and Schultz 1998; Fiorillo et al. 2003; Pan et al. 2005).

\section{Instrumental learning}

We measured DA efflux in rats learning that a lever press leads to pellet delivery, which involves contingency learning (Balleine and Dickinson 1998) in combination with other, i.e., Pavlovian processes (Parkinson et al. 2000; Dayan and Balleine 2002). Such Pavlovian mechanisms are reported to depend on DA transmission (Dickinson et al. 2000; Di Ciano et al. 2001), whereas learning about instrumental outcomes apparently does not (Dickinson et al. 2000; Dayan and Balleine 2002). The role of DA in the approaching (preparatory) phase of rewarddirected behavior might be explained in this way. In the learning group of the present experiment, the Pavlovian process of acquisition (Kelley et al. 1997), boosting (Cardinal et al. 2002) or stamping-in (Wise 2004) of incentive motivation apparently took place in the first session, as directly from the start of the second session, these rats show high levels of reward-oriented activities (nose pokes) and obtain almost the maximum number of pellets. The high DA increases and the difference between learning and nonlearning groups that we report in the first session may reflect this process. The data presented by Smith-Roe and Kelley (2000) suggest a causal relationship between activation of D1-receptors and instrumental learning, but do not differentiate between the underlying processes (see also Kelley 2004).

\section{DA and learning}

In general, our data are in line with previous findings, e.g., the DA measurements in the PFC reported for instrumental learning in rats by Izaki et al. (1998) and for avoidance learning in gerbils by Stark et al. (1999), who suggest activation selectively during acquisition of the task. Human microdialysis data (Fried et al. 2001) also suggest a higher activation of DA efflux (in the amygdala) during task acquisition. It is striking that these different examples relate to DA in PFC, NAC, and amygdala, different projection areas of the mesocorticolimbic DA system, suggesting a general involvement of the various projections of this system in task acquisition. 
The immediate consequence of the activation of DA is thought to be an increased attention and responsiveness to salient stimuli (Beninger 1983; Redgrave et al. 1999; Joseph et al. 2003). Other studies suggest that a stimulation of DA activity contingent upon an operant response potentiated cellular changes related to the operant behavior (Reynolds et al. 2001; Brembs et al. 2002). It is tempting to suggest that the extra activation of DA efflux that we observed in the learning group support these changes and the (probably related) protein-dependent consolidation of instrumental learning (Hernandez et al. 2002). Indeed, Baldwin et al. (2002a) reported that post-session administration of inhibitors of protein kinase A also inhibited learning (Baldwin et al. 2002a), indicating that $\mathrm{DA}_{1}$-receptor-mediated mechanisms initiated this process. Interestingly, Dalley et al. (2005) reported $\mathrm{D}_{1}$-receptor-dependent post-session consolidation of Pavlovian autoshaping, whereas Kelley (2004) mentions that this does not affect instrumental learning. Thus, acquisition, consolidation, and expression of Pavlovian approach behavior appear to depend on accumbal DA, whereas up to now, no such role for DA in contingency learning has been found. However, at this moment, a role for DA in the acquisition and performance of lever pressing as an action-outcome process cannot yet be dismissed. This role may be explained by the theories of Salamone et al. (2003), who propose that accumbens DA is involved in behavioral activation and in facilitation of resources to work toward a goal.

\section{Individual differences}

An intriguing finding in some previous studies was the relation between DA activation in PFC (Stark et al. 1999) or amygdala (Fried et al. 2001) and learning performance. We present evidence that DA increases in NAC are also related to individual differences in the acquisition of an instrumental task. We discussed the indications that the increases we observed may reflect the process of acquisition of incentive motivation (see above). If this is true, individual differences in DA activation during instrumental learning would reflect differences in either innate or acquired incentive motivation.

It is interesting to note that rats with enhanced levels of DA activity in the NAC may show a higher reactivity to novelty and a higher sensitivity to reinforcing properties of food (highresponders) than rats with lower levels of DA (low responders) (Dellu et al. 1996). Such an animal can be expected to make more nose pokes and to press an available lever more readily. However, preliminary data suggest that high responsivity to novelty was not related to the acquisition of lever pressing in Pavlovian autoshaping (Flagel et al. 2004). Further studies will be needed to show which factors make some rats acquire a simple instrumental learning task faster than others.

\section{Acknowledgments}

Part of this work was supported by Pfizer Central Research.

\section{References}

Baldwin, A.E., Holahan, M.R., Sadeghian, K., and Kelley, A.E. 2000. $\mathrm{N}$-Methyl-D-aspartate receptor-dependent plasticity within a distributed corticostriatal network mediates appetitive instrumental learning. Behav. Neurosci. 114: 1-15.

Baldwin, A.E., Sadeghian, K., Holahan, M.R., and Kelley, A.E. 2002a. Appetitive instrumental learning is impaired by inhibition of cAMP-dependent protein kinase within the nucleus accumbens. Neurobiol. Learn. Mem. 77: 44-62.

Baldwin, A.E., Sadeghian, K., and Kelley, A.E. 2002b. Appetitive instrumental learning requires coincident activation of NMDA and DA D1 receptors within medial prefrontal cortex. J. Neurosci. 22: 1063-1071.

Balleine, B.W. and Dickinson, A. 1998. Goal-directed instrumental action: Contingency and incentive learning and their cortical substrates. Neuropharmacology 37: 407-419.

Bassareo, V. and Di Chiara, G. 1997. Differential influence of associative and non-associative learning mechanisms on the responsivencess of prefrontal and accumbal dopamine transmission to food stimuli in rats fed ad libitum. J. Neurosci. 17: 851-861.

Bassareo, V., De Luca, M.A., and Di Chiara, G. 2002. Differential expression of motivational stimulus properties by dopamine in nucleus accumbens shell versus core and prefrontal cortex. $J$. Neurosci. 22: 4709-4719.

Beninger, R.J. 1983. The role of dopamine in locomotor activity and learning. Brain Res. Brain Res. Rev. 6: 173-196.

Berridge, K.C. and Robinson, T.E. 1998. What is the role of dopamine in reward: Hedonic impact, reward learning, or incentive salience? Brain Res. Brain Res.Rev. 28: 309-369.

Blackburn, J.R., Pfaus, J.G., and Phillips, A.G. 1992. Dopamine functions in appetitive and defensive behaviours. Prog. Neurobiol. 39: 247-279.

Brembs, B., Lorenzetti, F.D., Reyes, F.D., Baxter, D.A., and Byrne, J.H 2002. Operant reward learning in Aplysia: Neuronal correlates and mechanisms. Science 296: 1706-1709.

Cardinal, R.N., Parkinson, J.A., Hall, J., and Everitt, B.J. 2002. Emotion and motivation: The role of the amygdala, ventral striatum, and prefrontal cortex. Neurosci. Biobehav. Rev. 26: 321-352.

Cheng, J.J., de Bruin, J.P.C., and Feenstra, M.G.P. 2003. Dopamine efflux in nucleus accumbens shell and core in response to appetitive classical conditioning. Eur. J. Neurosci. 18: 1306-1314.

Dalley, J.W., Lääne, K., Theobald, D.E.H., Armstrong, H.C., Corlett, P.R., Chudasama, Y., and Robbins, T.W. 2005. Time-limited modulation of appetitive Pavlovian memory by D1 and NMDA receptors in the nucelus accumbens. Proc. Natl. Acad. Sci. 102: 6189-6194.

Dayan, P. and Balleine, B.W. 2002. Reward, motivation, and reinforcement learning. Neuron 36: 285-298.

Dellu, F., Piazza, P.V., Mayo, W., Le Moal, M., and Simon, H. 1996. Novelty-seeking in rats-Biobehavioral characteristics and possible relationship with the sensation-seeking trait in man. Neuropsychobiology 34: 136-145.

Di Chiara, G. 2002. Nucleus accumbens shell and core dopamine: Differential role in behavior and addiction. Behav. Brain Res. 137: $75-114$.

Di Ciano, P., Cardinal, R.N., Cowell, R.A., Little, S.J., and Everitt, B.J. 2001. Differential involvement of NMDA, AMPA/Kainate, and dopamine receptors in the nucleus accumbens core in the acquisition and performance of Pavlovian approach behavior. $J$. Neurosci. 21: 9471-9477.

Dickinson, A., Smith, J., and Mirenowicz, J. 2000. Dissociation of Pavlovian and instrumental learning under dopamine antagonists. Behav. Neurosci. 114: 468-483.

Feenstra, M.G.P. 2000. Dopamine and noradrenaline release in the prefrontal cortex in relation to unconditioned and conditioned stress and reward. Progr. Brain Res. 126: 133-163.

Feenstra, M.G.P. and Botterblom, M.H.A. 1996. Rapid sampling of extracellular dopamine in the rat prefrontal cortex during food consumption, handling and exposure to novelty. Brain Res. 742: $17-24$.

Feenstra, M.G.P., Botterblom, M.H.A., and Mastenbroek, S. 2000. Dopamine and noradrenaline efflux in the prefrontal cortex in the light and dark period: Effects of novelty and handling and comparison to the nucleus accumbens. Neuroscience 100: 741-748.

Fiorillo, C.D., Tobler, P.N., and Schultz, W. 2003. Discrete coding of reward probability and uncertainty by dopamine neurons. Science 299: 1898-1902.

Flagel, S., Robinson, T., and Akil, H. 2004. Individual differences in Pavlovian conditioned approach behavior. Abstract Viewer/Itinerary Planner, Program No. 671.12. Society for Neuroscience, Washington, D.C.

Fried, I., Wilson, C.L., Morrow, J.W., Cameron, K.A., Behnke, E.D., Ackerson, L.C., and Maidment, N.T. 2001. Increased dopamine release in the human amygdala during performance of cognitive tasks. Nat. Neurosci. 4: 201-206.

Hernandez, L. and Hoebel, B.G. 1988. Feeding and hypothalamic stimulation increase dopamine turnover in the accumbens. Physiol. Behav. 44: 599-606.

Hernandez, P.J., Sadeghian, K., and Kelley, A.E. 2002. Early consolidation of instrumental learning requires protein synthesis in the nucleus accumbens. Nat. Neurosci. 5: 1327-1331.

Hollerman, J.R. and Schultz, W. 1998. Dopamine neurons report an error in the temporal prediction of reward during learning. Nat. Neurosci. 1: 304-309.

Ikemoto, S. and Panksepp, J. 1999. The role of nucleus accumbens dopamine in motivated behavior: A unifying interpretation with special reference to reward-seeking. Brain Res. Brain Res. Rev. 31: 6-41.

Izaki, Y., Hori, K., and Nomura, M. 1998. Dopamine and acetylcholine 
elevation on lever-press acquisition in rat prefrontal cortex. Neurosci. Lett. 258: 33-36.

Joseph, M.H. and Hodges, H. 1990. Lever pressing for food reward and changes in dopamine turnover and uric acid in rat caudate and nucleus accumbens studied chronically by in vivo voltammetry. $J$. Neurosci. Methods 34: 143-149.

Joseph, M.H., Datla, K., and Young, A.M. 2003. The interpretation of the measurement of nucleus accumbens dopamine by in vivo dialysis: The kick, the craving or the cognition? Neurosci. Biobehav. Rev. 27: 527-541.

Kelley, A.E. 1999. Neural integrative activities of nucleus accumbens subregions in relation to learning and motivation. Psychobiology 27: $198-213$.

2004. Memory and addiction: Shared neural circuitry and molecular mechanisms. Neuron 44: 161-179.

Kelley, A.E., Smith-Roe, S.L., and Holahan, M.R. 1997. Response-reinforcement learning is dependent on

$\mathrm{N}$-methyl-D-aspartate receptor activation in the nucleus accumbens core. Proc. Natl. Acad. Sci. 94: 12174-12179.

McCullough, L.D., Cousins, M.S., and Salamone, J.D. 1993. The role of nucleus accumbens dopamine in responding on a continuous reinforcement operant schedule: A neurochemical and behavioral study. Pharmacol. Biochem. Behav. 46: 581-586.

Montague, P.R., McClure, S.M., Baldwin, P.R., Phillips, P.E.M., Budygin, E.A., Stuber, G.D., Kilpatrick, M.R., and Wightman, R.M. 2004. Dynamic gain control of dopamine delivery in freely moving animals. J. Neurosci. 24: 1754-1759.

Pan, W.-X., Schmidt, R., Wickens, J.R., and Hyland, B.I. 2005. Dopamine cells respond to predicted events during classical conditioning: Evidence for eligibility traces in the reward-earning network. J. Neurosci. 25: 6235-6242.

Parkinson, J.A., Cardinal, R.N., and Everitt, B.J. 2000. Limbic cortical-ventral striatal systems underlying appetitive conditioning. Prog. Brain Res. 126: 263-285.

Paxinos, G. and Watson, C. 1997. The rat brain in stereotaxic coordinates, 3rd ed., Academic Press, London.

Redgrave, P., Prescott, T.J., and Gurney, K. 1999. Is the short-latency dopamine response too short to signal reward error? Trends Neurosci.
22: $146-151$.

Reynolds, J.N.J., Hyland, B.I., and Wickens, J.R. 2001. A cellular mechanism of reward-related learning. Nature 413: 67-70.

Robbins, T.W. and Everitt, B.J. 1996. Neurobehavioural mechanisms of reward and motivation. Curr. Opin. Neurobiol. 6: 228-236.

Roitman, M.F., Stuber, G.D., Phillips, P.E.M., Wightman, R.M., and Carelli, R.M. 2004. Dopamine operates as a subsecond modulator of food seeking. J. Neurosci. 24: 1265-1271.

Salamone, J.D., Keller, R.W., Zigmond, M.J., and Stricker, E.M. 1989. Behavioural activation in rats increases striatal dopamine metabolism measured by dialysis perfusion. Brain Res. 487: 215-224.

Salamone, J.D., Correa, M., Mingote, S., and Weber, S.M. 2003. Nucleus accumbens dopamine and the regulation of effort in food-seeking behavior: Implications for studies of natural motivation, psychiatry, and drug abuse. J. Pharmacol. Exp. Ther. 305: 1-8.

Satoh, T., Nakai, S., Sato, T., and Kimura, M. 2003. Correlated coding of motivation and outcome of decision by dopamine neurons. $J$. Neurosci. 23: 9913-9923.

Schultz, W. 2002. Getting formal with dopamine and reward. Neuron 36: 241-263.

Smith-Roe, S.L. and Kelley, A.E. 2000. Coincident activation of NMDA and dopamine D1-receptors within the nucleus accumbens core is required for appetitive instrument learning. J. Neurosci. 20: $7737-7742$.

Sokolowski, J.D., Conlan, A.N., and Salamone, J.D. 1998. A microdialysis study of nucleus accumbens core and shell dopamine during operant responding in the rat. Neuroscience 86: 1001-1009.

Stark, H., Bischof, A., and Scheich, H. 1999. Increase of extracellular dopamine in prefrontal cortex of gerbils during acquisition of the avoidance strategy in the shuttle-box. Neurosci. Lett. 264: 77-80.

Wightman, R.M. and Robinson, D.L. 2002. Transient changes in mesolimbic dopamine and their association with 'reward'. J. Neurochem. 82: 721-735.

Wise, R.A. 2004. Dopamine, learning and motivation. Nat. Rev. Neurosci. 5: $483-494$.

Received August 19, 2005; accepted in revised form January 2, 2006. 


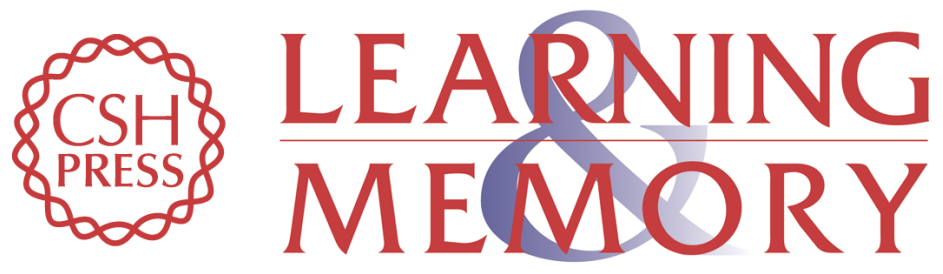

\section{Individual differences in dopamine efflux in nucleus accumbens shell and core during instrumental learning}

Jingjun Cheng and Matthijs G.P. Feenstra

Learn. Mem. 2006, 13:

Access the most recent version at doi:10.1101/lm.1806

References This article cites 49 articles, 14 of which can be accessed free at:

http://learnmem.cshlp.org/content/13/2/168.full.html\#ref-list-1

License

Email Alerting Receive free email alerts when new articles cite this article - sign up in the box at the Service top right corner of the article or click here. 